Revue d'histoire de l'enfance " irrégulière »

Le Temps de l'histoire

$6 \mid 2004$

Les sciences du psychisme et l'enfance « irrégulière »

\title{
Les destins liés de la figure du "psy" et de l'appareil de l'enfance inadaptée
}

Alain-Noël Henri

\section{(2) OpenEdition \\ 12 Journals}

Édition électronique

URL : http://journals.openedition.org/rhei/835

DOI : $10.4000 /$ rhei.835

ISBN : 978-2-7535-1644-1

ISSN : $1777-540 \mathrm{X}$

Éditeur

Presses universitaires de Rennes

Édition imprimée

Date de publication : 15 novembre 2004

Pagination : 219-249

ISSN : 1287-2431

Référence électronique

Alain-Noël Henri, «Les destins liés de la figure du "psy" et de l'appareil de l'enfance inadaptée », Revue d'histoire de l'enfance « irrégulière » [En ligne], 6 | 2004, mis en ligne le 31 mai 2007, consulté le 03 décembre 2020. URL : http://journals.openedition.org/rhei/835; DOI : https://doi.org/10.4000/rhei. 835 


\section{Les destins liés de la figure du "psy" et de l'appareil de l'enfance inadaptée}

La façon dont une société nomme et traite ceux qui sont porteurs de ce qu'elle tente de refouler - et qui lui revient sous les traits insupportables d'une inquiétante étrangeté -, est l'une des meilleures voies d'accès à la compréhension de l'organisation symbolique qui est sa substance même. Lorsque Foucault décrit magistralement comment l'âge classique ${ }^{(2)}$ inscrit la folie dans les repères de la moralité rationnelle tout en l'enfermant derrière des murs - en nombreuse compagnie -, il met le doigt sur un fait de société global qui déborde largement son objet initial.

De même, la fin du xxème siècle, non sans soubresauts encore perceptibles aujourd'hui, voit s'épanouir un autre modèle dans lequel les catégories de la santé se substituent à celles de la moralité, tandis que se substitue à l'enfermement le maintien d'une présence dans le tissu social, sous la garantie et la surveillance d'une nuée d'experts présumés. Entre les deux, on peut suivre l'émergence progressive du second modèle, assorti de conflits souvent aigus avec le premier, mais aussi et surtout d'une succession de formations de compromis souvent ingénieuses.

Deux de celles-ci prennent un relief particulier, parce qu'elles furent à la fois inaugurales et emblématiques, et qu'elles se traduisirent par la mise en place de deux appareils sociaux d'ampleur impressionnante : la médecine aliéniste, de la fin du XviIIème siècle à la fin du Xxème, et l'enfance inadaptée, à partir, en gros, de la deuxième guerre mondiale. Nous allons essayer de suivre comment cette dernière fut, entre autres, concomitante de l'apparition d'un nouveau personnage, que nous appellerons comme on l'appelle le plus couramment, le "psy", concept dont l'extension est finalement mieux déterminée que ceux qu'il recouvre ou recoupe, tels que le psychiatre, le psychologue, le psycho-

\section{Alain-Noël Henri $^{(1)}$}

(1) Maître de conférences honoraire à l'université Lumière Lyon 2.

(2) En fait, la société bourgeoise artisanale à son apogée, une fois clos son antagonisme avec la société féodale-rurale et avant l'ouverture de la société industrielle.

Alain-Noël Henri / p. 219 à 249 
thérapeute ou le psychanalyste, entre lesquels le bon peuple, d'ailleurs, peine à se retrouver.

\section{Aux origines de l'enfance inadaptée}

\subsection{Un autre regard sur l'enfance}

\subsubsection{De l'enfant justiciable de sa famille à l'enfant victime de sa famille}

Il est piquant de constater que, dans les deux cas, l'entrée en scène de ces nouveaux appareils fut ponctuée d'un épisode mettant en jeu et en cause l'appareil judiciaire, et sur un scénario assez comparable.

Dans le premier tiers du XIXème siècle, la médecine engage avec l'appareil judiciaire et policier une partie de bras de fer dont l'enjeu est l'appropriation du fou criminel. "Il est criminel, il est à moi ", dit l'une en substance. "Il est fou, il est à moi ", dit l'autre. L'histoire est connue, et l'on sait qu'elle se conclura par le pouvoir somme toute exorbitant conféré à l'expertise médicale de prononcer l'irresponsabilité pénale, non point formellement en lieu et place du procureur ou du juge, mais de fait en interposant en amont de leur jugement (au sens logique du terme) un jugement préjudiciel pratiquement indiscutable.

C'est ce scénario qui se réitère, de part et d'autre de la seconde guerre mondiale, autour cette fois de l'enfance coupable. Bien entendu, on sait que, quand l'histoire se répète, elle bégaie, et les deux processus ont différé sensiblement sur certains aspects. À l'origine, par exemple, ce ne fut pas cette fois la caste savante qui en appela à l'opinion publique ; il serait plus juste de dire qu'elle ramassa finalement la mise d'un processus résultant initialement d'un mouvement d'opinion orchestré par des producteurs d'idéologie "généralistes", journalistes ou écrivains à succès.

Il est toujours artificiel de dater un début, mais il me semble qu'on peut retenir comme premier acte de cette mutation la campagne d'Alexis Danan contre les «bagnes d'enfants ", à la suite de la révolte de la colonie pénitentiaire de Belle-Île-en-Mer en 1934. Dans les vingt années qui suivirent, de nombreuses œuvres de fiction jouent sur ce même ressort d'une substitution de l'apitoiement et de la sollicitude à la vindicte hai- 
neuse : poèmes (la Chasse à l'enfant, de Jacques Prévert), romans (Chiens perdus sans collier, de Gilbert Cesbron), essais (Graine de crapules, de Fernand Deligny), films (Los Olvidados, de Luis Buñuel).

En ce premier temps, le ressort n'est pas encore la substitution, à une figure de coupable, d'une figure de malade, mais bien celle d'une figure de victime. Certes l'assignation à la maladie comme l'assignation à la place de victime ont en commun d'annuler a priori toute imputation, en exilant le sujet de sa position de sujet, à laquelle est substitué le statut de produit d'une cause. Néanmoins, la différence n'est pas mince, et elle prend même une singulière actualité en un temps où reviennent en force les figures de la victimisation.

Le statut particulier du mineur par rapport à la justice pénale n'est pas alors une nouveauté. Le Code pénal, dès 1810, la loi de 1850 instituant les colonies pénitentiaires, celle de 1912 instituant les tribunaux pour enfants et la liberté surveillée ont jalonné une histoire qu'il serait aisé de réinterpréter a posteriori comme l'émergence progressive du regard contemporain sur l'enfance coupable. Illusion d'optique assurément, car le mineur n'y est pas caractérisé par une irresponsabilité pénale. On ne peut comprendre ces différentes mesures que si l'on réalise l'importance de la coupure qui sépare à l'origine, en droit pénal, le correctionnel du criminel. Les auteurs de crimes encourent l'affliction et l'infamie. Les auteurs de délits sont susceptibles de correction. La "maison de correction " est d'ailleurs restée dans la mémoire collective comme le lieu d'enfermement des enfants, alors qu'elle est d'abord le lieu d'exécution des peines correctionnelles.

Le mineur apparaît, dans ce contexte, comme exonéré seulement d'imputation criminelle, même lorsque ses agissements relèvent de la définition légale d'un crime. Il est en revanche pleinement "correctionnalisé", même s'il est jugé avoir agi sans discernement, à ceci près qu'il relève fondamentalement de la juridiction paternelle, dans l'espace privé de la famille, et subsidiairement de la juridiction publique représentée par la justice correctionnelle. À voir comment, dans les dictionnaires du XIXème siècle, la notion de correction est fortement associée à la puissance paternelle, on peut même se demander si ce n'est pas le juge correctionnel qui est, même pour les adultes, investi d'une mission 
(3) Faute d'un concept à la fois admis et opératoire pour désigner l'ensemble des manifestations qui attaquent l'ordre symbolique d'une société et enclenchent en retour des pratiques visant à en réduire l'impact, j'ai proposé ailleurs le terme de “mésinscription”. Faute de place ici pour déplier ce concept, je me contenterai de l'épithète "troublante", qui a le mérite de révéler que le concept médical de “trouble”, dès lors qu'il est appliqué non à une fonction biologique mais à un comportement social, sert essentiellement à déplacer ce qu'il désigne en fait, c'est-à-dire le trouble, au sens le plus banal, produit sur l'environnement social du sujet présumé "troublé".

(4) Ou du moins de celui de ses rameaux, très prégnant dans cette de père, plus que le père d'une mission de juge, alors que la justice criminelle, la seule d'ailleurs, en France, à être rendue par un jury populaire symbolisant le transfert au peuple des pouvoirs régaliens, serait plutôt héritière du châtiment divin.

La figure de l'enfant victime, qui vient introduire un nouvel équilibre dans le second tiers du Xxème siècle, n'est certes pas non plus nouvelle mais, au XIXème siècle, elle s'attachait en priorité à l'enfant privé d'une famille, orphelin ou abandonné, et livré de ce fait aux soins d'étrangers malintentionnés. Cosette, Jane Eyre, Oliver Twist, Remi (le héros de Sans famille), autant de figures pathétiques d'être exposées à tous les dangers faute de parents pour les protéger - sans même parler, plus lointainement, des héroïnes de contes livrées à la méchanceté d'une marâtre. Le lieu d'enfermement de l'enfance troublante, ${ }^{(3)}$ c'est d'ailleurs " l'orphelinat ", où les orphelins stricto sensu ne sont plus depuis belle lurette majoritaires, s'ils l'ont jamais été.

La nouveauté est que la figure désormais prégnante est celle de l'enfant victime de sa famille. Victime éventuellement de mauvais traitements : ainsi les "comités Alexis Danan ", constitués dans la lancée de la campagne de presse sus-évoquée, ont-ils longtemps perduré autour de la traque obsédante des enfants battus et demeurent-ils l'un des points d'appui majeurs du thème de l'enfance maltraitée. Mais ce thème-là n'est pas vraiment non plus une nouveauté ; il inspire déjà manifestement la loi de 1890. Ce qui est neuf dans l'imagerie qui apparaît alors, c'est plutôt celui de l'enfant victime d'un manquement de la famille (et non plus d'un manque de famille), soit que la défaillance provienne de sa dissolution, soit qu'elle s'impute au manque d'amour (maternel) et, secondairement, d'autorité (paternelle).

\subsubsection{Donner les moyens de pousser plutôt que remettre droit}

Alors se développe tout naturellement le double paradigme des enfants du divorce et des carences affectives. Cette dernière expression est intéressante pour notre propos : elle est évidemment savante, et, provenant de la lignée psychanalytique, ${ }^{(4)}$ elle pourrait passer pour signer l'entrée en scène d'une thématisation psychologique. Mais c'est à peine 
si, à cette étape, on peut vraiment y lire son influence. Il serait plus exact de dire qu'elle vient là fournir une caution savante à une idée vulgaire, celle d'une privation d'amour maternel.

C'est en fait - à partir de ses marges, comme il advient souvent - le regard sur l'éducation qui change ici de nature. Ce qui s'annonce ainsi à propos de l'enfance inquiétante, c'est d'ailleurs moins l'apparition d'une nouveauté absolue que l'inversion d'un rapport de dominance, qui conduit à la généralisation et au primat de figures attestées antérieurement, mais marginalement.

Depuis le XviIIème siècle, l'éducation est un champ de tension entre deux idéologies antagonistes. D'un côté, l'idéologie orthopédique : une matière (au mieux informe, au pire vicieuse dès l'origine) qui tend à dévier spontanément de la norme (c'est-à-dire étymologiquement de la rectitude de la règle), et qu'il s'agit de « mettre droit ». De l'autre côté, l'idéologie rousseauiste : une bonne nature qu'il suffit de cultiver et de laisser se développer intelligemment. Jusque-là, la balance avait toujours penché en faveur de la première. Au milieu du xxème siècle, la seconde commence à prendre le pas.

On voit là que la vieille terminologie du dressage, du redressement et de la correction, rejointe ultérieurement par l'extension du préfixe ortho, ne doit rien au hasard. Tout converge vers le « mettre droit ». A contrario, le basculement en faveur de l'idéologie rousseauiste met au premier plan le paradigme de la plante, et par là de son corollaire, le développement. L'éducateur forgeron, qui martèle et redresse de toute la force de ses muscles, fait place à l'éducateur jardinier, qui observe avec patience, veille à fournir les nutriments lorsque la terre est en défaut et protège les jeunes pousses fragiles de la violence des agressions extérieures. Protéger et nourrir : ici se noue le lien entre le modèle carentiel et le thème qui ultérieurement deviendra central, celui de la protection de l'enfance. L'enfant troublant n'est plus porteur d'un « quelque chose en trop » qu'il s'agit de contraindre, mais d'un «quelque chose en moins » auquel il s'agit de suppléer.

L'enjeu déborde largement la question de l'enfant : c'est bien du conflit entre deux modèles de rapports sociaux qu'il s'agit, l'un fondé sur une hiérarchie pyramidale où les niveaux supérieurs doivent déter- période, qui interprète la psychanalyse comme fondement d'une orthopédie psychique. 
(5) Cette mesure exceptionnelle ne relevait d'ailleurs pas à l'origine de ce qu'on appelle aujourd'hui la « protection de l'enfance ». Avant la loi de 1889, l'article du Code pénal qui la prévoit ne figure pas dans la partie consacrée aux « crimes et délits envers l'enfant », mais dans celle qui vise « les attentats aux mœurs ", à titre de peine accessoire réprimant l'incitation à la débauche des mineurs. Son objet n'est pas de soustraire l'enfant à un danger, mais de punir un délinquant en le privant d'un de ses droits fondamentaux. Même si la loi de 1889 s'inscrit en revanche clairement dans une optique de protection de l'enfance, les mesures de déchéance resteront relativement rares. miner exactement les niveaux inférieurs, l'autre fondé sur un réseau dynamique où les interactions et les rétroactions entre des sources créatives indépendantes produisent globalement un équilibre satisfaisant. On comprend aisément comment la moralité fait référence majeure dans le premier cas, et la santé, avec à l'arrière-plan le paradigme biologique, dans le second.

\subsubsection{Deux compromis de transition}

Dans le parallélisme entre les deux épisodes, le plus intéressant est peutêtre dans leur fonction de transition. Tous deux en effet illustrent le fait que, comme on l'a signalé en débutant, les mutations historiques se signalent d'abord sous des formes qui, a posteriori, apparaissent comme des compromis, en ce qu'elles introduisent des nouveautés qui se révèleront ultérieurement nodales, tout en conservant des aspects non moins nodaux de l'organisation antérieure. Cet aspect n'est pas assez souvent mis en valeur, et cela tend à brouiller considérablement la lisibilité de ces formations de transition.

Ainsi, la psychiatrie asilaire scandait bien le passage d'une société fondée sur la moralité rationnelle, selon les canons de l'humanisme, à une société fondée sur les catégories de la santé, selon les canons de la scientificité. Mais elle conservait en même temps l'enfermement comme mode de traitement de l'inquiétante étrangeté. De même, dans le nouveau regard sur l'enfance inquiétante, on voit bien que la famille est toujours représentée sous la forme caricaturale d'une double fonction de protection dévolue à la mère et d'autorité dévolue au père. Mais, d'une part, sa défaillance est désormais banalisée, intégrée dans le champ du socialement représentable, alors qu'elle n'était antérieurement prise en compte que sous les formes extrêmes et quasi monstrueuses justifiant la déchéance de la puissance paternelle. ${ }^{(5)} \mathrm{D}$ 'autre part, l'ordre de priorité s'est inversé, la référence maternelle primant désormais sur la référence paternelle. Enfin et surtout, c'est une imagerie du manque à combler qui vient se substituer à l'imagerie de la déformation à corriger. 


\subsection{Un autre regard sur l'inquiétante étrangeté}

\subsubsection{La médicalisation, un processus moins simple qu'il n'y paraît}

Il serait, on l'a dit, erroné de voir dans l'apparition de la notion de carence affective l'effet d'une allégeance à un point de vue psychologique (elle ne doit d'ailleurs pas grand'chose à une problématisation proprement psychanalytique). Elle fait en revanche très authentiquement lien avec la pensée médicale. Elle transpose en effet purement et simplement le concept de carence alimentaire, dont l'avitaminose fournit le paradigme central. Elle est donc le chaînon qui vient rattacher initialement la figure de la victime à la figure du malade. Ce glissement est d'une importance décisive dans la genèse de l'appareil de l'enfance inadaptée. Et, en effet, cette mutation du regard sur l'enfance, qui se signale à partir des marges, rencontre le mouvement parallèle d'assignation de l'inquiétante étrangeté aux catégories de la santé. Elle le rencontre d'autant plus aisément qu'il traduit exactement la même chose dans un autre registre.

$\mathrm{Si}$, dans un premier temps, l'appareil social de la médecine joue ici le même rôle central qu'un siècle et demi plus tôt autour de la folie, c'est d'abord, tout simplement, qu'il était seul disponible, dans l'organisation antérieure, comme dépositaire des catégories de la santé. En cela aussi, l'histoire est donc bien une réitération : de nouveau s'impose le regard proprement médical comme substitut du regard moral.

Mais à y bien regarder, le parallélisme n'est pas si clair. Il y a du trompe-l'œil dans cet aspect-là de la répétition, même si la psychiatrie, point d'aboutissement de la médecine aliéniste et point de départ de l'appareil de l'enfance inadaptée, semble marquer une évidente continuité. Car ce n'est pas de la même psychiatrie qu'il s'agit, et pas seulement parce que plus d'un siècle sépare les deux histoires. L'une et l'autre ont certes en commun d'utiliser le cheval de Troie de la sollicitude philanthropique pour introduire la médecine dans la citadelle de l'appareil répressif. Mais la comparaison ne va guère plus loin. Ce n'est pas du même usage social de la médecine qu'il s'agit.

Si l'on se reporte cinquante ans en arrière, on ne peut qu'être frappé en effet de l'hiatus qui sépare alors de la psychiatrie d'adultes cette psychiatrie de l'enfant encore naissante. ${ }^{(6)}$ On s'étonne même que les deux

(6) Ou plutôt de la neuropsychiatrie infantile : la première chaire de psychiatrie de l'enfant ne fut créée pour Heuyer qu'en 1948. La psychiatrie infantojuvénile prendra plus tard le relais, et le mot pédopsychiatrie est encore beaucoup plus tardif. 
espaces aient pu se développer si différemment à partir du même personnel, formé dans les mêmes filières, régi par les mêmes textes et uni dans le même statut social.

Bien mieux, nous verrons plus loin que les développements qu'inaugure l'intervention de la médecine se marqueront notamment, à la sortie de ces formes de transition, par l'apparition d'autres appareils sociaux, animés par d'autres personnages, fonctionnant selon d'autres modèles idéopraxiques, et que son monopole initial sera dès lors battu en brèche par des formes certes apparentées, mais néanmoins différentes et concurrentes (y comprise d'ailleurs la néopsychiatrie qui émerge progressivement dans le droit fil de la sectorisation).

Cette diversification des figures dépositaires des catégories de la santé est en partie masquée par les effets de la coupure sociale forte, juridiquement étayée par tout un arsenal de textes de droit public, entre ce qui est réputé appartenir à la médecine et ce qui est réputé être dehors - coupure secondairement assouplie, mais aussi obscurcie par la catégorie frontière du paramédical. Il y a bien entendu une fonction à la radicalité de cette coupure : il n'en reste pas moins que, si l'on s'efforce de la mettre entre parenthèses, d'autres distributions apparaissent qui la recoupent en de multiples parts. Il en va des frontières juridiques entre les appareils sociaux comme des frontières entre les états : elles coïncident souvent avec de réelles frontières sociologiques, mais elles sont aussi parfois des artefacts masquant une géographie sociale plus complexe.

\subsubsection{Mutation dans la psychiatrie}

Alors que par ailleurs l'appareil asilaire a perduré pendant un siècle et demi, il faudra moins d'une génération pour que prenne fin l'emprise exclusive de la médecine stricto sensu sur les nouveaux espaces de traitement de l'enfance. C'est que la maturation de la grande substitution d'un modèle de rapports sociaux à un autre est alors beaucoup plus avancée. Nous allons essayer de préciser un peu les jalons de ce processus.

Ce que faisait la médecine aliéniste naissante en s'emparant du fou criminel, c'était de proposer ses propres murs pour assurer la protection de la société contre le danger qu'il représente, comme garants aussi sûrs 
que les murs de la prison (voire même que la guillotine), tout en l'exonérant d'un soupçon d'injuste cruauté à l'égard de ces "infortunés » que sont les "déments ».

En revanche, le nouveau regard médical sur l'enfance coupable, qui se rattache pour l'essentiel à la psychiatrie infantile telle qu'elle se développe à partir des années vingt ${ }^{(7)}$ autour de Georges Heuyer, s'alimente d'une tout autre source : celle du courant hygiéniste qui déjà, dans la seconde moitié du XIXème siècle, s'organisait autour du triptyque " pauvreté maladies infectieuses (et notamment tuberculose) - immoralité » sur un fond de préoccupations essentiellement sociales. Le même mouvement, qui retournait les " classes dangereuses » en victimes d'un manque (la pauvreté) engendrant le double fléau de la maladie et de l'immoralité et qui préparait en cela l'assimilation de la seconde à la première, se retrouve ici appliqué à l'enfance dangereuse.

Même si le hiatus est loin d'être comblé aujourd'hui, de nombreuses anastomoses se sont tissées depuis les années soixante-dix pour reconstituer un continuum entre psychiatrie adulte et pédopsychiatrie. C'est en fait que la seconde a été rejointe par la première dans une évolution qu'elle anticipait. En réalité, l'enfant apparaît ici comme l'un des principaux objets emblématiques d'une néopsychiatrie qui commence, pendant ces deux décennies d'entre-deux-guerres, à se détacher du bloc idéopraxique généré par l'appareil asilaire. Ce n'est d'ailleurs pas à partir des espaces d'enfermement qu'elle esquisse sa singularité, mais comme il advient souvent des nouveautés historiques, à partir d'un petit nombre d'espaces périphériques, notamment de "l'infirmerie spéciale des aliénés au dépôt de la préfecture de Police ", ce fief de Clérambault qui vit passer, comme internes ou comme médecins, des figures aussi marquantes et contrastées que celles d'Heuyer, Lacan ou Le Guillant. Heuyer lui-même s'est intéressé à bien d'autres objets que l'enfance, l'alcoolisme par exemple. Inversement, des praticiens comme Le Guillant, Bonnafé ou Tosquelles, figurent simultanément au premier plan d'entreprises visant les adultes, comme le centre de traitement et de réinsertion sociale de Villejuif, et d'autres visant les enfants.

Dans ce bouillonnement, les repères idéologiques sont pour le moins brouillés. On y voit coexister nosographie classique, psychanalyse,
(7) C'est aussi la période où le mot aliéniste achève de devenir obsolète. 
modèles traditionalistes d'ordre moral et social associés à une dénonciation de l'exploitation sociale qui rassemblera une partie importante de ce courant dans les rangs du parti communiste (bien que la référence proprement marxiste soit là, au minimum absente, au maximum présente à titre d'habillage).

Sur le versant infantile, est en outre incorporée dans la sauce la lignée " médico-pédagogique " inaugurée par Binet à partir des "arriérés d'école », avec une extension passablement acrobatique aux « arriérés d'asile»: elle fournissait un compromis bien utile entre ces deux contraires que sont le modèle du redressement orthopédique (la " rééducation ") et celui du rétablissement d'un développement naturel contrarié (avec notamment l'offre d'un terrain privilégié aux différentes familles de la "pédagogie nouvelle " réduites dans l'enseignement ordinaire au statut de minorités isolées). Ajoutons-y un zeste de pavlovisme par-ci par-là (" déconditionner pour reconditionner »). Je crains d'en oublier, tant le mélange fut riche et confus, le plus surprenant, et donc le plus instructif, étant que cet éclectisme semblait ne pas gêner grand monde.

\subsubsection{Une relative neutralité idéologique}

Il a été du coup aisé, selon ce qu'on voulait démontrer, d'imputer ensuite cette effervescence, soit à un progressisme profitant de la Libération pour s'épanouir après avoir fourbi ses armes dans la Résistance, soit au contraire à un ordre moral quasi maurrassien profitant du régime de Vichy pour asseoir durablement son emprise. Ne cherchons pas à trancher : les deux sont vrais. Tosquelles, Le Guillant, le mouvement des communautés d'enfants illustrent la première thèse. La mise en place par le régime de Vichy du réseau des associations de sauvegarde de l'enfance et de l'adolescence quadrillant le territoire illustre la seconde. Il n'en reste pas moins que non seulement il y a eu cohabitation et synergies entre les deux filiations après 1945, mais encore qu'on recherche vainement entre les deux des différences de pratiques et de repères vraiment significatives. Tout juste peut-on observer que, autour de la très laïque Association nationale des communautés d'enfants, un bloc fortement marqué par le Parti communiste s'ancrait essentiellement autour de l'Éducation natio- 
nale, tandis que l'Union nationale des associations régionales de sauvegarde de l'enfance et de l'adolescence, où dominait l'héritage confessionnel, prenait plus appui sur un réseau de neuropsychiatres notabilisés, dont chacun était empereur en sa région, tels que Lafon à Montpellier ou Kohler à Lyon. Mais, sur le terrain, le système était de fait aussi politiquement neutre qu'il était théoriquement éclectique.

Cette association d'un apolitisme présumé à un éclectisme théorique n'est pas anecdotique. Il dénote une évidence implicite quasi fondatrice, qui ne sera remise en cause que brièvement dans l'après 68 , selon laquelle la réponse au malheur exclut le travail de la contradiction. Il peut certes y avoir du débat supposé technique sur l'efficacité des réponses, mais l'unanimité sur les enjeux ne souffre aucune mise en cause. L'objectivité présumée du "besoin" et l'impératif catégorique de sa satisfaction verrouillent solidement cette armature idéologique qui, bien entendu, est battue en brèche de toute part dans la réalité concrète, aucun espace social réel ne pouvant échapper aux effets de contradiction.

\section{L'enfance inadaptée, des années d'après-guerre aux années soixante-dix}

\subsection{Le melting pot}

\subsubsection{Convergence et agglutination}

L'appareil de l'enfance inadaptée se développe dans l'après-guerre à la fois par "croissance externe" et par "croissance interne". C'est-à-dire qu'on voit à la fois de nouvelles institutions fleurir à un rythme plus que soute$\mathrm{nu}$, et d'autres, beaucoup plus anciennes, et parfois très anciennes, venir s'agglomérer à ce noyau. Dans les deux cas, ce sont des filiations multiples et passablement disparates qui viennent $y$ brasser leurs gènes.

Il y a d'abord celles contre lequel le nouvel appareil vient s'inscrire, mais dont il hérite sous bien des aspects : le système pénitentiaire, et en particulier les "colonies agricoles ", mais aussi la prison (au début des années soixante les établissements d'éducation surveillée comportaient encore un « mitard » par exemple). De même, progressivement s'y intégreront les espaces asilaires, moins ceux que la psychiatrie continue à gérer que ceux 
dévolus à ces " arriérés profonds » qu'au XIXème siècle la médecine aliéniste avait refusé d'endosser.

L'internat scolaire fournit aussi un important apport, d'autant que sa fonction géographique tend rapidement à disparaître à mesure des progrès de l'urbanisation et de l'explosion démographique de l'enseignement secondaire, qui resserre le maillage territorial des établissements, faisant passer au premier plan la population des enfants "mis en pension" et des placements à caractère social. L'orphelinat opère sa mue au cours des années soixante, en grande partie du fait de la crise culturelle, démographique et économique des congrégations religieuses, contraintes de faire appel aux financements publics et à des personnels laïcs. Enfin, plus marginales et moins connues, de très anciennes institutions pour aveugles et sourds (les plus anciennes en fait en matière de pédagogie spéciale, puisqu'elles remontent à l'abbé de l'Épée et à Valentin Haüy, donc à la fin du XVIIIème siècle) tombent à leur tour dans l'escarcelle.

En regard, on trouve les modèles au nom desquels sont battus en brèche ces espaces traditionnels et qu'on a déjà évoqués au passage. D'abord l'hôpital, ou plutôt l'établissement médical privé. Puis l'enseignement dit « spécial ", car si, jusqu’à la loi de 1975, l’Éducation nationale conserve jalousement une cloison étanche avec ses classes de perfectionnement, qui se multiplient d'ailleurs en cette période, ses modèles ne s'exportent pas moins dans le nouvel espace, à la fois pour l'organisation des temps dits "scolaires " et surtout en ce qui concerne la grille de lecture psychométrique des "déficiences ». C'est l'âge d'or du QI.

On a d'autre part signalé comment tous les courants de la pédagogie nouvelle, qu'ils se réferent à Freinet, à Decroly, à Montessori, voire à l'anthroposophie, ont colonisé massivement ces espaces, les seuls avec l'école maternelle à ne pas les ignorer superbement. On ne saurait enfin sousestimer l'apport des mouvements de jeunesse, notamment de la branche "extension" du scoutisme et de la JOC, ainsi que du mouvement d'éducation populaire issu du Front populaire et amplifié par la Résistance.

Plus marginalement, mais leur influence a dépassé leur poids numérique, s'y sont ajoutés des espaces d'accueil pour les enfants sans famille, bien distincts des anciens orphelinats, quelques-uns issus d'initiatives 
individuelles remontant à l'avant-guerre, mais la plupart liés à la guerre ellemême avec son cortège de persécutions massives et de déplacements de populations. On en distingue deux grands rameaux : les « républiques d'enfants ", organisées autour d'un modèle politique de citoyenneté démocratique, et les familles de substitution, attachées à reconstituer les fonctionnements d'une famille "normale " à l'intérieur d'une institution collective, soit autour de couples mariés, soit autour d'éducatrices célibataires, fédérées en général autour d'un fondateur ou d'une fondatrice charismatique.

\subsubsection{Nouveaux espaces}

Le système invente par ailleurs des espaces originaux, qui surgissent en des points très divers du champ de forces créé par ce processus d'agglutination. Les uns marquent une étape décisive dans la mise en cause du modèle jusque-là hégémonique de l'enfermement, les autres un remaniement supplémentaire dans la figure de l'enfant victime.

Jusque-là, nous n'avons rencontré que des pratiques d'internat. Comme l'asile d'aliénés un siècle plus tôt, le nouveau système commence donc par conserver ce qui avait été pendant trois siècles au cœur même des pratiques sociales de la déviance : l'enfermement. Du moins en apparence, car en réalité ces collectivités d'enfants des années cinquante s'organisent selon un compromis original, qu'on pourrait nommer l'espace « fermé-ouvert ». Ouverture surtout symbolique, marquée notamment par celle, voyante et quasi provocante, du portail d'entrée. Mais aussi amorce d'une réelle ouverture, avec des signes discrets : liens des enfants et des adolescents avec l'environnement, surtout en milieu rural, cas de la majorité de ces institutions ; traitements spécialisés, voire scolarisation à l'extérieur ; traitement moins répressif de la fugue, qui se transforme parfois en « fugue officielle » (l'enfant est dûment déclaré en fugue, mais l'on sait où il est et le lien éducatif est maintenu avec lui)...

Bien plus radicale, et annonciatrice du grand basculement des pratiques sociales qui se développe dans les années soixante et soixante-dix au nom d'un même mot d'ordre "faites tomber les murs", est l'apparition, d'une part du milieu ouvert avec ses différentes variantes (dérivé il est vrai en partie de la pratique du service social), d'autre part de la pré- 
vention spécialisée, issue d'initiatives bénévoles qui surgissent simultanément sur tout le territoire, là aussi autour de personnages charismatiques, dans l'approche informelle des bandes de jeunes délinquants.

L'autre surgissement majeur résulte de ce nouvel événement fondateur qu'est la constitution, dans le Rhône, de la première association de parents d'enfants inadaptés, très rapidement imitée dans de nombreux départements et bientôt dans tous, l'ensemble étant non moins rapidement fédéré en une puissante organisation nationale, l'UNAPEI (Union nationale des associations de parents et d'amis d'enfants inadaptés).

Ce nouveau "sous-appareil", outre son importance quantitative - il se révèle d'une impressionnante fécondité dans la création d'établissements divers -, révèle et accélère la maturation du thème de l'enfant-victime, prolongeant, tout en en prenant sur un point essentiel le contre-pied, le mouvement qui avait en premier retourné la figure de l'enfant coupable. D'une part, il fait monter en puissance un paradigme de l'enfant inadapté concurrent - vite victorieux - de ce dernier : l'enfant débile, puisque c'est encore à l'époque ainsi qu'on le nomme. Plus besoin pour celui-là de retourner un "trop-plein" en manque : le manque est sa définition même, mesuré par un ratio (le QI, bien sûr...) dont bien peu d'esprits impertinents s'aventurent alors à contester l'objectivité et l'immutabilité. D'autre part, il invalide radicalement le thème de l'enfant victime de sa famille, puisque bien au contraire la famille est ici, en quelque sorte, "co-victime".

\subsubsection{Les éléments unificateurs}

On devine aisément qu'un tel melting-pot aboutit, dans les premières décennies à une extraordinaire variété. Trois éléments ont été décisifs pour engendrer une dynamique d'unification : d'abord, dans le registre des idées, le concept même d'inadaptation ; ensuite, dans celui des acteurs, l'émergence du métier d'éducateur spécialisé ; enfin, dans le registre institutionnel, un type de structure bien oublié aujourd'hui, mais qui a représenté entre 1945 et 1970 le cœur du dispositif : les centres d'observation.

Premier unificateur, donc, le concept d'inadaptation. La filiation sémantique est évidente. Le verbe "adapter » est fort ancien, mais l'extension à l'adaptation sociale est, à l'époque qui nous occupe, encore 
très récente : si le Larousse du Xxème siècle illustre encore, en 1931, le dérivé "inadapté » (ignoré à ma connaissance de tous les dictionnaires du XIXème siècle) par l'expression "l'élimination automatique des inadaptés ", on ose espérer que le rédacteur n'a en tête que les espèces animales en stricte théorie darwinienne... Il n'en est que plus significatif de voir ce quasi-néologisme remplacer en quelques années l'ensemble des vocables mis à l'essai au fil des décennies précédentes (enfance anormale, troublée, irrégulière, etc.). Quelle était donc sa vertu singulière ?

Dans ce genre d'épisode lexical, un vocable doit en général sa fortune à son pouvoir de condensation, lui permettant de masquer d'un pont de neige la faille d'une contradiction. Je fais l'hypothèse que le terme " inadaptation " doit la sienne à sa double racine, mécanique et biologique, répondant aux deux figures implicites de l'éducateur forgeron et de l'éducateur jardinier. Elle parvient en effet à gommer leur opposition.

"Ces excentriques sont ajustables": cette admirable sentence que Boris Vian emprunte à un ouvrage technique pour la mettre en exergue d'un des chapitres de L'Automne à Pékin, nous introduit au cœur de la métaphore mécanique. Tout en conservant le pouvoir démiurgique du praticien bien outillé, elle déplace en effet l'image du « redressement " vers, d'une part, une image topologique (le rapport entre un centre et une périphérie, qu'on retrouvera plus tard avec la marginalité), d'autre part une image de modulation fine de la forme, dont la défectuosité est rapportée à une inadéquation locale et conjoncturelle et non plus à l'universalité de la ligne droite. On ne ramène plus en force à une normalité canonique, on use patiemment redents et surépaisseurs pour harmoniser les pièces d'un ensemble. L'anglais maladjusted, par lequel se traduit usuellement inadapté, renvoie directement à cette métaphore. Le forgeron se rapproche du jardinier.

Inversement, la référence biologique que draine le rameau darwinien module sérieusement l'optimisme rousseauiste de la pédagogie nouvelle, à peu près dans le même mouvement qui a substitué à la même époque le dogme keynésien au dogme de l'économie libérale. Certes le paradigme demeure celui d'un développement endogène opposé à celui d'une conformation exogène. Mais le darwinisme met en lumière que l'efficacité du laisser-faire repose sur l'impitoyable élimination des tentatives ratées. 
Aussi, de même que la crise de 1929 a installé l'idée que les effets des crises cycliques du capitalisme sont un prix à payer excessif en regard des bénéfices de leur effet sélectif, ce qui contraint à superposer des correctifs volontaristes au libre jeu de la concurrence, de même l'idée d'inadapté, faute de pouvoir soutenir celle de leur élimination naturelle, convoque-t-elle l'idée de suppléer à leur faiblesse par ces mêmes correctifs volontaristes.

Ainsi, tandis que le rassemblement dans un même appareil institutionnel d'une culture de l'internat et d'une culture du milieu ouvert masque l'antagonisme majeur concernant l'inquiétante étrangeté, le concept d'inadaptation masque l'antagonisme majeur concernant l'enfance. Le nouveau système est moins le haut lieu d'un modernisme que l'espace d'une confrontation feutrée entre traditionalisme et modernisme.

Deuxième unificateur : le métier, nouveau, d'éducateur spécialisé. Il résulte de la conjonction entre deux processus à l'œuvre, l'un au niveau de l'offre d'emploi, l'autre au niveau de l'offre de pratique.

L'offre d'emploi n'était au départ que la conséquence mécanique de l'internat. Il n'y avait là rien de bien nouveau : tous les espaces d'enfermement depuis le XviIème siècle ont suscité une main-d'œuvre subalterne vouée à la surveillance et au gardiennage, main-d'œuvre peu abondante (parce que les taux d'encadrement étaient à l'origine très faibles), peu payée (ou même payée en gratification identitaire, comme la maind'œuvre religieuse), peu considérée, et sans formation, le plus souvent recrutée dans une main-d'œuvre locale où prédominent les origines rurales. Dans les institutions préexistantes qui s'agglutinent dans le nouvel appareil, cette main-d'œuvre préexiste. Dans nombre d'institutions qui se créent, ce recrutement continue, et cette population n'est pas au départ ciblée comme acteur autonome : c'est aux fondateurs, médecins, enseignants, bénévoles issus de la bourgeoisie aisée ou d'organisations militantes, qu'appartient le champ de pratique.

Ces derniers appartiennent en effet à une population tout autre : leur origine sociale, leur parcours universitaire et/ou leur expérience militante, leur conferent une efficacité sociale importante, décuplée par les opportunités qu'ouvre à l'innovation sociale la période agitée et féconde de 
l'après-guerre. Et ils disposent d'un modèle qui contrebalance puissamment celui du surveillant et du gardien : celui du mouvement de jeunesse ou de la colonie de vacances. Ceux-ci ont secrété en effet, depuis quelques décennies, un nouveau type de personnage, le moniteur et surtout le chef scout. On mesure mal aujourd'hui la puissance et la cohérence de ce qu'a été cette culture des mouvements de jeunesse et d'éducation populaire, recoupant elle aussi transversalement les clivages politiques et idéologiques les plus tranchés.

Toutefois, entre ces militants propulsés parfois très jeunes dans le statut de notable et les figures essentiellement juvéniles pour ne pas dire adolescentes proposées par ces nouveaux modèles, se glisse une faille douloureuse : comment faire prendre au sérieux ce métier qui sera longtemps suspect d'être un métier de gamins jouant avec des gamins ? Dans chaque internat, on trouvait toujours un enfant pour demander à un éducateur : "Et toi, qu'est-ce que tu feras plus tard comme métier ?"

Par ailleurs, la représentation d'une pratique éducative active liée au changement de regard sur l'enfance oblige à améliorer le taux d'encadrement, crée donc un appel d'air en termes quantitatifs. Très vite, dès 1943, se sont créées quelques écoles d'éducateurs spécialisés (Montesson, Lyon, Montpellier). Celles-ci sont encore loin d'être porteuses d'une culture professionnelle propre : juxtaposition d'une formation "théorique ", elle-même juxtaposition de cours magistraux disjoints dispensés par des spécialistes externes où prédominent médecins et juristes, et d'une formation "pratique » où prédominent les techniques occupationnelles, mais aussi et surtout les stages. Presque simultanément, se crée l'Association nationale des éducateurs de jeunes inadaptés (l'ANEJI), dont le rôle sera essentiel dans la métabolisation de cette nouvelle profession.

Il est d'ailleurs symbolique que le premier texte contractuel sur les conditions de rémunération et d'emploi du secteur de l'enfance inadaptée ait été signé, en 1958, entre l'UNARSEA (qui regroupe au niveau national les ADSEA) et l'ANEJI. Mais à mesure que les écoles mettent de nouveaux diplômés sur le marché, cette couche d'éducateurs "notabilisés”, dont certains siégeaient d'ailleurs dans les deux organismes signa- 
(8) L'acronyme SEA pour Sauvegarde de l'enfance et de l'adolescence - se retrouve dans les ADSEA (associations départementales), les ARSEA (associations régionales, disparues au moment de la création des CREAI), l'UNARSEA (ou, par abréviation UNAR, Union nationale des associations régionales), le SNASEA (syndicat national). De même, l'acronyme APEI se retrouve décliné dans les URAPEI, l'UNAPEI et le SNAPEI.

(9) Ou plus exactement le « centre de rééducation ", puisque c'est alors l'appellation qui prévaut. taires, cèdent la place à des personnels nettement plus "prolétarisés", qui fondent des syndicats affiliés aux grandes confédérations. On mesure le chemin parcouru quand on retrouve pour signer, huit ans après seulement, la première convention collective du secteur, autant de syndicats de salariés que de grandes centrales, tandis que le SNASEA, ${ }^{(8)}$ fondé pour la circonstance par l'UNARSEA, doit partager son monopole avec le SNAPEI (créé par l'UNAPEI) et le SOP (Syndicat des œuvres privées, créé par l'UNIOPSS Union nationale des œuvres privées sanitaires et sociales).

De ces débuts, on retiendra deux choses ; d'abord ce qualificatif «spécialisé ", qui a souvent été ensuite l'objet de plaisanteries multiples tant ce spécialiste apparaît depuis toujours comme un généraliste, Maitre Jacques occupant les postes de travail les plus variés auprès d'objets de pratique qui ne le sont pas moins. Il s'agissait bien entendu de le distinguer de l'éducateur en général, mais après tout un éducateur sportif ou artistique aurait aussi bien mérité l'appellation. En fait, la référence, qu'on retrouve aussi dans « l'enseignement spécialisé » ou plus tard dans «l'hôpital spécialisé » (comprenez " psychiatrique "), vise évidemment l'objet de cet éducateur, cet objet "spécial ", avec la même connotation que lorsqu'on dit « c'est spécial » d'un plat au goût dérangeant, ou d'un livre ou d'un film par trop transgressif.

On retiendra ensuite que, lorsque l'ANEJI veut, en une sorte de manifeste, proclamer l'éminente dignité de cette fonction suspecte de n'être qu'affaire de très jeunes gens, elle forge cette expression qui servira longtemps de cri de ralliement : un "technicien des relations humaines". Proclamation qui n'est pas sans rappeler la définition de l'étudiant par l'UNEF, dans la même année 1947, comme " jeune travailleur intellectuel ", sorte de revendication auto-persuasive parlant d'une douloureuse faille identitaire. Spécialiste sans spécialité et technicien sans technicité : une étrange profession venait de naître.

Troisième unificateur : le centre d'observation. Point de passage obligé vers l'internat spécialisé,(9) ou éventuellement les services de milieu ouvert, ce type particulier d'internat accueille, en général pendant trois mois, tous ceux que le verdict du juge d'enfants ou du neuropsychiatre infantile désigne au statut d'enfant ou d'adolescent «inadapté ». L'organisation 
s'inspire à l'évidence du séjour d'observation à l'hôpital. La vie quotidienne y est exactement la même que dans les internats. Mais en outre, sous la direction d'un médecin psychiatre qui opèrera la synthèse finale, l'enfant est l'objet, d'une part d'observations systématiques de tous ceux qui l'encadrent, éducateurs et instituteurs, selon des grilles détaillées, d'autre part d'examens psychiatrique et psychologique ; enfin sa situation familiale fait l'objet d'un rapport de l'assistante sociale.

\subsection{Handicap et protection de l'enfance :}

\section{la bipolarisation du système}

Dès lors que la figure du débile était venue faire pièce à celle de l'enfant " coupable-excusé ", la bipolarité entre l'imputation à une famille défaillante et l'imputation à la mauvaise fortune ne cessera plus d'organiser en profondeur le nouvel appareil social, la première tendant à l'autonomiser par rapport au regard médical, et la seconde à l'y arrimer.

Cette bipolarisation a relégué au second plan l'usage du terme "inadapté ", sans pour autant que ce que cette appellation dénotait ait jamais été invalidé. Elle se structure à la suite de deux processus de nature très différente qui, au cours des années soixante, ont fortement marqué le nouveau système.

\subsubsection{L'enfant en danger}

Le premier est de nature juridique et par suite institutionnelle : il résulte de l'ordonnance de décembre 1958. On sait que celle-ci, en incorporant dans le Code civil (et non plus pénal) les dispositions aujourd'hui connues de tous concernant les mineurs dont « la santé, la sécurité, la moralité ou l'éducation " sont jugées en danger, met le point d'orgue à l'évolution qui, depuis la fin du XIXème siècle, met en place progressivement la notion de protection de l'enfance. Il existe déjà entre les deux guerres, par exemple, une Inspection générale de la protection de l'enfance.

L'évolution est parallèle à celle de la notion d'enfant victime. Le devoir de protection de l'État s'exerce en premier à propos de ses pupilles, enfants privés de famille par le décès ou l'abandon. Puis il s'étend à l'enfance en " danger moral ", avec toute l'ambiguité du terme, qui sert à la fois à 
étendre le danger au-delà du "danger physique ", mais qui fait aussi évidemment référence à la norme de moralité. La nouveauté de l'ordonnance de 1958, c'est que cette notion jusque là subsidiaire devient le cœur du dispositif d'ordre public concernant l'enfance troublante.

\subsubsection{Le handicap}

Le second processus est terminologique. Il résulte de l'apparition du terme " handicapé » et de son succès non moins fulgurant, vers le milieu des années soixante, que celui, vingt ans plus tôt, du terme "inadapté ". Curieux est le destin de cette métaphore hippique, puisque le cheval handicapé est celui qui est par nature trop bien armé dans la compétition ; s’il y a mise en œuvre d'un correctif volontariste, c'est à l'extrémité supérieure de la distribution. Or, étrangement, le mot se met à s'appliquer par extension, comme le vocable "inadapté ", à ceux qui sont les moins bien armés dans la compétition, le handicap étant non plus le correctif, mais le processus « naturel » qu'il s'agit de contrebalancer.

Dans la filiation sémantique, le terme est d'abord venu remplacer « l'infirme » et "l'invalide » pour désigner celui qui n'a pas la pleine disposition de son corps, et en particulier de ses membres, de ses jambes pour se déplacer et de ses mains pour travailler : l'infirme et l'invalide sont au principal une figure d'adulte, c'est-à-dire d'un travailleur potentiel, à qui son exclusion de l'emploi n'est pas imputable à faute. Cette figure de l'invalide, vieil objet de compassion, s'était enrichie après la guerre de 1914 d'une connotation de dette vis-à-vis des invalides de guerre, et il est remarquable que la plupart des mesures en faveur des invalides civils aient été des extensions de mesures qui avaient été d'abord des compensations pour les victimes de la guerre. Ainsi s'est établie progressivement la notion d'un dî̀ à leur égard.

Pour ma part, c'est dans le cadre de l'Association nationale des communautés d'enfants (ANCE), fortement liée à l'Éducation nationale, que j'ai pour la première fois, en 1965 ou 1966, rencontré cette proposition de substituer le label du handicap à celui de l'inadaptation, d'abord pour les déficients mentaux, mais aussi bien pour les " cas sociaux ». Le terme trahissait l'ambivalence de la communauté enseignante vis-à-vis de la compéti- 
tion, et, corrélativement, de l'égalité : car elle est prise entre le thème de la restauration d'une compétition ne prenant en compte que les capacités et le mérite (l'inégalité étant quasiment synonyme de ce qu'en économie on nomme " distorsion de concurrence") - ce qui conduit l'instruction publique à noter et classer en permanence les élèves -, et une extension généralisée de l'égalité des chances qui récuse la notion même de compétition. La notion de handicap s'introduit ici comme un heureux compromis, qui maintient le primat de la compétition tout en annulant ses effets à la limite inférieure de la distribution des capacités.

\subsubsection{Le couple bipolaire}

S'il est question ici de bipolarisation, c'est que le doublet « enfant en danger »/« enfant handicapé » ne fonctionne pas comme une nouvelle partition de l'ensemble préalablement rassemblé par la notion d'inadaptation. L'appareil de traitement, avec ses lieux, ses acteurs, ses rituels, ses discours, demeure un système sociologiquement unifié. Mais les imageries de la protection de l'enfance et du handicap, et l'oscillation qui s'établit entre elles au gré des fantasmes divers suscités par l'objet inquiétant, traduisent un dépliement de la contradiction dont les termes se condensaient dans la notion d'inadaptation.

Cette bipolarité déborde l'espace de l'enfance, comme l'atteste la réorganisation administrative essentielle qu'est la création, en 1964, aux niveaux départemental et régional, de directions unifiées de l'action sanitaire et sociale. Ainsi s'inaugure un couple sémantique, le "sanitaire et social ", qui, sans coïncider avec le couple " handicap-enfance en danger ", renvoie à une distribution symbolique connexe. L'enfance est d'ailleurs le noyau organisateur de ce nouvel espace. C'est en effet en même temps et dans la même visée stratégique, sous l'impulsion du même haut fonctionnaire, Bernard Lory, que les associations régionales de Sauvegarde de l'enfance et de l'adolescence, cadre devenu trop étroit puisque débordé de toute part par d'autres associations, deviennent les CREAI (centres régionaux de l'enfance et de l'adolescence inadaptées). 


\section{L'émergence des "psys"}

Nous voici maintenant à pied d'œuvre pour resituer dans ce contexte la question qui fait l'objet de cet article et de l'ensemble dans lequel il s'inscrit : comment les "psys" ont-ils été introduits dans le nouvel appareil social?

\subsection{La place originelle de la psychiatrie}

\subsubsection{D'abord seule de son espèce}

On a déjà vu la forte implication de la psychiatrie dès son origine, et plus précisément de ce rameau passablement isolé alors au sein de la psychiatrie, malgré son alliance avec les courants les plus modernistes, qu'était la neuropsychiatrie infantile, alliée aux courants modernistes de la psychiatrie générale. Quant aux autres catégories intriquées qui constituent aujourd'hui la nébuleuse des "psys", la plupart n'existent pas encore, ou seulement à l'état naissant.

Le mot psychothérapie ne désigne encore qu'un ensemble de pratiques appartenant en propre à la psychiatrie. Seule la psychanalyse peut revendiquer une existence distincte, dès lors qu'elle est théoriquement ouverte aux non-médecins. Encore cette ouverture restera-t-elle longtemps fort restreinte, en particulier en France, où la psychanalyse avait pourtant été introduite par une polonaise non médecin, Eugénia Sokolnicka, accueillie à bras ouverts par Heuyer à Sainte-Anne. Mais, victimes d'une assimilation à l'intérêt, jugé suspect, des milieux littéraires et artistiques, et en particulier des surréalistes, pour le freudisme, les non-médecins avaient vu leur accès à l'institution psychiatrique barrée par un oukase catégorique d'Henri Claude. Et, même si la Société psychanalytique de Paris ne leur est pas vraiment fermée, elle restera longtemps le garant sourcilleux de l'arrimage de la psychanalyse à la médecine.

Quant à la psychologie, elle est loin d'être déjà un métier, même s'il existe déjà des psychologues : il s'agit plutôt encore d'une qualification seconde liée à la fréquentation d'une discipline intellectuelle enseignée depuis un demi-siècle, et surtout à la maitrise de la méthode des tests, qui s'est imposée depuis l'entre-deux-guerres comme l'outil caractéristique du psychologue praticien. Il faudra attendre le milieu des années cinquante 
pour que, à la suite de la création en 1947 de la licence de psychologie et de la clarification progressive du statut des diplômes d'université - qui avaient fleuri antérieurement dans le désordre et qui deviennent des titres de qualification professionnelle post-licence -, des psychologues dûment labellisés arrivent vraiment sur le marché du travail.

\subsubsection{Une emprise importante, mais à nuancer}

Il faut toutefois préciser la nature de l'emprise de la psychiatrie sur l'appareil naissant de l'enfance inadaptée. Elle est écrasante au niveau du contrôle des personnes morales gestionnaires de statut privé, notamment des présidences d'association, et, ce qui va de pair, au niveau de la crédibilité auprès des pouvoirs publics. Lorsque les fondateurs ne sont pas eux-mêmes médecins, il leur est, dans les premiers temps, très difficile de mener à bonne fin leurs projets sans l'actif soutien d'un médecin. Bref, dans le vivier des notables, sans le "patronage" desquels, depuis le début des temps modernes, aucune initiative privée à visée de régulation sociale n'est possible, le dépositaire d'une présumée technicité scientifique en rapport avec les catégories de la santé a ici détrôné sans équivoque le bourgeois philanthrope, comme lui-même avait jadis évacué le grand seigneur bien en cour.

À l'intérieur même des institutions, le tableau est plus contrasté. Dans le centre d'observation, si la vie quotidienne est sans partage sous contrôle éducatif, la fonction de triage et de ventilation est clairement sous contrôle médical et organisée selon les modèles de la procédure diagnostique en milieu hospitalier, les différents membres de l'équipe curieusement dénommée "pluridisciplinaire " (dont bien peu de membres représentent une "discipline " stricto sensu) étant assimilés à des auxiliaires médicaux, faisant rapport à un psychiatre responsable de la synthèse et du verdict final.

Dans les institutions à visée éducative, en revanche, le poids du psychiatre décroît progressivement à mesure qu'on se déplace sur une échelle qui irait du "médical " au "social ». À une extrémité, le modèle est celui de la clinique spécialisée à direction médicale et à personnel infirmier ou assimilé à du personnel infirmier. À l'autre extrémité, les 
clubs et équipes de prévention sont pratiquement sans référence médicale, même si un médecin, le Dr Flavigny, figure au premier plan de la galerie des fondateurs.

Entre les deux, tout dépend du rapport singulier qui s'établit entre le directeur, éventuellement les équipes, et le psychiatre vacataire, auquel les textes d'agrément, en particulier bien entendu lorsque le payeur est la Sécurité sociale, font obligation d'avoir recours. Il peut aussi bien être le gourou, dont la parole fait autorité indiscutable, qu'une couverture purement formelle, dont l'influence sur les repères symboliques dominants dans l'établissement est quasiment nulle, avec toutes les nuances imaginables entre les deux.

\subsubsection{Les allégeances idéologiques}

Si cette place de la psychiatrie est de plus en plus floue à mesure que l'on descend des échelons hiérarchiques les plus élevés, dont la fonction est d'assurer l'interface avec la société globale, vers les échelons subalternes, qui assurent l'interface avec la réalité concrète de l'enfance troublante, c'est que, comme on l'a vu, la neuropsychiatrie infantile de l'époque est plus dépositaire d'un empilement éclectique de repérages théorico-idéologiques que porteuse de la culture psychiatrique dont elle est issue : tout simplement parce que l'impressionnant appareil nosographique construit au cours du siècle qui précède par la psychiatrie d'adultes n'est que très marginalement applicable à l'enfance. Il parait aujourd'hui incroyable, par exemple, que le concept de « psychose infantile ", devenu si central, ne soit pratiquement pas utilisé avant la fin des années cinquante. L'enfant fou ne sera d'ailleurs que très progressivement incorporé dans les cohortes de l'enfance inadaptée : lorsqu'il n'est pas purement et simplement renvoyé derrière les murs de l'hôpital, et lorsqu'il ne se laisse pas ramener aux catégories dominantes de " caractériel " ou de " débile ", il y est l'objet du même regard terrifié que dans la société globale. Si étrange que cela puisse paraitre, le psychiatre ne règne pas sur l'appareil de l'enfance inadaptée comme gardien de la folie, mais comme une sorte de missionnaire qui, depuis sa terre d'origine (l'asile de fous), entreprend la conquête de l'ensemble des espaces de stigmatisation sociale. 
En face du tout-puissant psychiatre, le personnage du psychologue est non seulement rare, mais encore confiné dans son rôle de grand ordonnateur des tests. En revanche, la psychologie comme « discipline savante " commence à tenir une place non négligeable dans la pensée éducative au cours des années soixante. Si tant est qu'on puisse parler de «la " psychologie, tant sont hétéroclites les discours qui s'abritent sous cet auvent. La caractérologie, par exemple, dont l'existence semble aujourd'hui presque oubliée, mais dont la vogue dans le grand public fut grande il y a cinquante ans, fournit souvent la pensée classificatoire que la nosographie laisse vacante, avec l'avantage de ne pas être connotée par la présomption de pathologie. Mais, parmi les discours catalogués comme psychologiques, deux ont un destin plus particulier.

D'abord, dans les zones dominées par l'Éducation nationale, ou chez les éducateurs qui en proviennent, la psychologie génétique est une grande inspiratrice, avec des références dominantes successives à Wallon, auréolé de son appartenance au Parti communiste, puis à Piaget, qui, des années soixante aux années quatre-vingts, va incarner «la " psychologie pour plusieurs générations d'enseignants. D’autre part, la psychologie des groupes, fer de lance de l'introduction de la psychologie sociale américaine dans la culture française, notamment autour de l'ARIP, va fournir aux éducateurs spécialisés la référence " technique » qui manque tant à ces " techniciens de la relation", pour qui les «techniques éducatives " servant à occuper et à faire jouer les enfants sont un bien piètre champ de compétence pour se faire reconnaitre de l'opinion publique savante. Après les cadres de l'industrie, ils seront parmi les meilleurs clients des séminaires de dynamique de groupe.

Reste la place particulière de la psychanalyse. On a vu plus haut que c'est par le biais de la «carence affective » qu'elle fait son entrée dans les références de l'enfance inadaptée. Plus largement, mais dans la même lignée, elle est invoquée à l'appui d'une sorte de théorie anthropologique simplifiée, dans laquelle Freud aurait sans doute peiné à reconnaître les concepts-clés d'inconscient et de sexualité. Il ferait beau voir qu'on s'avise de penser l'enfance à travers le concept de sexualité infantile. Les dissidents qui gomment ou effacent l'accent sur la sexua- 
lité, tels qu'Adler ou surtout Jung, y connaissent d'ailleurs une vogue non négligeable. C'est en fait l'affectivité qui vient dans cette histoire se glisser à la place du sexuel. Si l'Eedipe n'est pas ignoré, il est presque systématiquement rabattu sur l'idée simple que l'enfant ne peut se " développer " qu'en présence de ses deux parents, et que chacun doit être conforme aux stéréotypes respectifs de la mère affectueuse et du père autoritaire. De servir d'habillage à l'hypothèse carentielle permet au vocabulaire psychanalytique de se glisser, lentement mais continûment, dans le vocabulaire quotidien des éducateurs et, à défaut de technicité, de contribuer à leur conférer une teinture de scientificité.

\subsection{Le tournant de 1968}

L'ébranlement inauguré par mai 68, s'il a été dans beaucoup de domaines une parenthèse, a véritablement, non pas produit, mais marqué un tournant essentiel dans l'ensemble de l'appareil de régulation sociale : ce qui s'est traduit, entre autre, sociologiquement, par un développement considérable et une forte structuration de ces nouvelles couches moyennes qui prennent appui sur ce qu'il est convenu d'appeler les "professions intermédiaires ». Jusque-là, seuls les enseignants du primaire, et dans une moindre mesure du secondaire, en constituaient les gros bataillons, sous d'autres appellations d'ailleurs.

\subsubsection{Le « socioculturel " et le " médico-social» entre sociologie et psychologie}

Traduisant une véritable révolution dans le fondement même des pratiques sociales, ce développement est particulièrement visible dans deux nouveaux espaces : le "socio-culturel ", héritier direct des mouvements d'éducation populaire, et le «médico-social », au cœur duquel on trouve l'appareil de l'enfance inadaptée.

Or ces deux champs de pratique sont indissociables du développement explosif simultané, et surtout de la popularisation dans le grand public, de deux champs de discours jumeaux, rassemblés sous l'auvent des "sciences humaines et sociales" : la sociologie et la psychologie - qui n'étaient pas pour rien à l'épicentre du mouvement étudiant. Si la pre- 
mière servit clairement de discours d'appui dominant au vaste appareil du « socio-culturel », la palme fut plus disputée au départ pour l'appareil médico-social. La thématique politique dérivée de mai 68 faisant, dans ce secteur dont on a vu plus haut le caractère constitutivement apolitique, une entrée tonitruante, les sociologues militants de cette génération y eurent une audience importante. Mais, si celle-ci put sembler égaler un temps celle de la psychologie, elle ne la dépassa jamais, et il ne fallut que quelques années pour que la balance se mette à pencher lourdement en faveur de cette dernière.

\subsubsection{La nouvelle naissance du psychologue praticien}

En tout état de cause, leurs modes d'inscription respectifs n'étaient pas du tout identiques. La sociologie est restée purement discursive. Elle n'a pu influer les champs de pratique que par trois accès : la référence livresque ; la formation (initiale, et à partir de 1971, continue) ; et ce qu'on appellerait aujourd'hui l'audit, sous les espèces de l'analyse institutionnelle.

En revanche, le tournant de 68 est celui d'une nouvelle naissance de la psychologie, qui va accentuer et asseoir son statut de pratique autonome, conditionné par un ensemble de modèles idéopraxiques cohérents. Notons tout d'abord que le mouvement de 68 est coextensif d'une véritable explosion démographique des étudiants en psychologie (la cohorte qui a commencé ses études en 1967 et qui était en première ligne en mai 1968 était trois fois plus nombreuse que celle qui l'avait précédée, et ce n'était qu'un début). Quelques années plus tard, cette génération avait marginalisé numériquement l'ensemble de ses prédécesseurs et imposé irrésistiblement ses propres modèles.

Elle a commencé par balayer le paradigme du psychologue-testeur, et plus globalement la restriction de la fonction au verdict du diagnostic. Cette position radicale ne tiendra pas très longtemps, la psychométrie et les épreuves projectives reprendront ensuite une place, mais devenue subalterne et accessoire. Car, entre temps, le noyau identitaire de la profession s'est déplacé sur la position de recours face à la difficulté psychique. Position qui se décline selon trois lignes principales : celle de thérapeute 
en relation duelle ; celle de " conseiller du prince " par rapport aux cadres et notamment aux directeurs des institutions ; et celle de soutien des équipes.

\subsection{L'émergence du "psy"}

\subsubsection{Un nouvel organisateur symbolique :}

\section{la souffrance psychique}

Ce dont on s'avise peut-être difficilement aujourd'hui, tant la chose paraît devenue évidente, c'est précisément que cela implique la constitution comme objet social majeur de la souffrance psychique en tant que telle. La stigmatisation de l'anomalie comme relevant des catégories de la santé avait certes déjà mis au premier plan la confusion, dans l'idée de maladie, du dysfonctionnement et de la souffrance. Mais ce qui est nouveau est l'inversion du rapport entre ces deux termes. Rétablir une fonction troublée et réduire la souffrance au passage n'est pas du tout la même visée que réduire la souffrance par le détour du rétablissement de la fonction troublée.

Cette novation majeure a pour corollaire la possibilité socialement offerte, pour la première fois, à celui qui intervient pour réduire le trouble, de s'identifier à la souffrance de l'objet porteur du trouble (l'objet " troublé-troublant "). L'enchaînement de signifiants qui sous-tend cet aspect de l'après-68 va de l'irruption d'une classe juvénile (en fait d'une adolescence indéfiniment prolongée) à son identification à la marginalité, et de là à la possibilité d'une revendication possible au nom de la souffrance psychique. Cet enchaînement rencontre le processus historique qu'on a longuement évoqué ci-dessus, dans lequel l'appareil de l'enfance inadaptée se constitue comme avant-garde d'un mouvement beaucoup plus ample et qui débordera vite le champ de l'enfance, sous l'invocation d'une réparation due à la victime, non plus essentiellement d'une maltraitance physique ou d'une inégalité économique, mais d'une douleur psychique.

Cela se traduit très concrètement par une explosion simultanée, d'une part de l'offre d'emploi en direction de ces nouveaux experts ès souffrance psychique que sont les infirmiers psychiatriques (profession autonomisée à cette époque, et ultérieurement réincorporée dans la profession infir- 
mière généraliste), les éducateurs et les psychologues ; et d'autre part de la demande d'emploi dans ces professions de la part de la population juvénile qui venait de se rassembler dans l'identification à la marginalité.

\subsubsection{Alliance et partage entre la néopsychiatrie et la psychologie}

C'est dans ce contexte que s'instaure entre psychiatrie, psychologie, psychothérapie et psychanalyse le système de rapports complexes et ambigus que nous évoquions en débutant, et qui, là encore, est particulièrement visible dans l'appareil de l'enfance inadaptée, décidément paradigme central du nouveau regard social sur l'objet troublant. Cette complexité vient de l'entrecroisement de plusieurs lignes de partage : entre des cultures professionnelles, des modes de formation, des filières de recrutement très différents ; entre des courants idéologiques héritiers du XIXème siècle et les positions modernistes qu'on a évoquées plus haut ; enfin entre des appareils sociaux différents. Il serait trop long de la débrouiller ici : on n'en retiendra que quelques lignes de force, au risque d'être suspect, aux yeux des familiers du milieu, de simplification abusive.

Dans l'appareil psychiatrique, ces années soixante-dix marquent le triomphe de la sectorisation, fer de lance de la vague néopsychiatrique, sur la pratique asilaire. L'entrée massive des psychologues s'y analyse comme une recherche d'alliances de la part des jeunes psychiatres modernistes, responsables de la création de leurs postes. En retour, ils concèdent aux psychologues, avec enthousiasme ou réticence, une part de leur position de notabilité. Mais ils demeurent, dans cet espace-là, totalement maitres du jeu, les psychologues n'étant pas en mesure de faire valoir d'autres points de vue que ceux qu'ils peuvent partager avec les médecins.

Dans l'appareil de l'enfance inadaptée, le rapport est très différent. Certes la fonction de garant vis-à-vis de la société globale reste dévolue au psychiatre, dépositaire encore incontesté du savoir sur les catégories de la santé étendues hors du champ de la médecine somatique. Mais, à l'intérieur des institutions, le psychologue, dont les temps de présence sont statistiquement beaucoup plus importants et dont la proximité sociale avec les éducateurs est surtout beaucoup plus grande, occupe le plus souvent une position-clé, comme supposé dépositaire du savoir. 
Outre que la psychologie bénéficie de la double focalisation sur la folie et sur l'enfance, qui la caractérise depuis la fin du XIXème siècle.

\section{Pour conclure : entre continuité et discontinuité, l'hésitation des catégories de la santé}

Mais peu importe en vérité quel rapport prévaut désormais entre psychologue et psychiatre dans l'appareil de l'enfance inadaptée : car entre temps s'est peu à peu accomplie, et en premier lieu dans ce creuset, la fusion symbolique que nous annoncions en débutant ; dans cette figure du "psy" qui devient, on l'a vu, la seule catégorie symbolique vraiment opératoire.

Ce qui est déterminant dans cette figure, qui tendra ensuite à s'imposer de plus en plus comme garante autonome des catégories de la santé en tant qu'organisateur de l'appareil de régulation sociale, et finalement mieux que la médecine elle-même, c'est qu'entre l'objet troublant et son environnement social elle introduit de la continuité là où la médecine, et plus encore la médecine asilaire, introduisait de la radicale discontinuité. Pendant un siècle et demi, la médecine asilaire avait été constituée gardienne d'une frontière étanche entre la normalité et l'horreur monstrueuse de la folie. Le "psy" est au contraire l'occupant d'une zone incertaine où l'objet troublant oscille entre cette altérité absolue et une proximité dans laquelle sa souffrance est miroir, grossissant et déformant certes, mais miroir quand même de la souffrance de tout un chacun. Corrélativement, il cautionne des pratiques non moins oscillantes entre deux extrêmes : la tentative d'emprise sur un objet chosifié et l'écoute d'un sujet. Le rapport a priori à l'étiologie organique et, par conséquent, à la pharmacodynamique fournira un excellent indicateur des aléas de cette oscillation. Un autre résidera dans la tension entre les modèles du "traitement " et les modèles de "l'aide ", tous deux condensés dans la polysémie du signifiant " soin ".

Dans cette histoire, deux signifiants vont gagner d'être propulsés à un statut symbolique central. L'un, du côté des "soignés", est la psychose ; l'autre, du côté des "soignants", est la psychanalyse, ou plutôt une nébuleuse de doctrines et de pratiques qui fleurissent autour d'elle et l'en- 
globent, souvent en marquant violemment à son égard une hostilité qui cache mal la fascination qu'elle exerce sur elles. La psychose, que les années soixante-dix instituent dans la même place où le XviIIème siècle avait érigé la statue du "bon sauvage ", parce qu'elle est le point de tension le plus extrême entre l'horreur méconnaissable et la secrète familiarité. La psychanalyse, parce qu'elle a été, dans l'histoire, la première à formaliser cette ambiguiité entre "l'autre » de la maladie et le "même " de la souffrance psychique, et qu'elle a fourni l'appareil conceptuel le plus cohérent et le plus riche pour en élaborer les contradictions.

Ainsi, parce qu'il s'est institué au carrefour de deux évolutions majeures, concernant l'enfance d'une part, l'inquiétante étrangeté d'autre part, qui peuvent s'analyser comme deux déclinaisons dans des champs différents de la même mutation, l'appareil de l'enfance inadaptée a-t-il été au foyer d'une histoire qui le déborde, comme l'a attesté d'ailleurs dans la suite l'extension de son champ bien au-delà de la coupure de la majorité civile. Et, si l'émergence des "psys" s'y est déployée avec plus d'aisance que partout ailleurs, c'est qu'elle a été elle-même une déclinaison de la même histoire. 